\title{
Prenatal ultrasound biometry related to subsequent blood pressure in childhood
}

\author{
K V Blake, L C Gurrin, L J Beilin, F J Stanley, G E Kendall, L I Landau, J P Newnham
}

J Epidemiol Community Health 2002;56:713-718

See end of article for authors' affiliations

Correspondence to:

Dr K V Blake, PMB 202

Alice Springs, Northern

Territory, Australia 0872;

laois26@hotmail.com

Accepted for publication

21 January 2002

\begin{abstract}
Study objective: To relate measures of fetal growth/size other than birth weight with subsequent blood pressure measured on the same individuals within the context of the "fetal origins of adult disease".

Design: A prospective cohort study in which measurements of fetal dimensions obtained by serial ultrasound imaging between 18 and 38 weeks gestation were analysed with reference to systolic blood pressure measurements on the offspring at age 6 years.

Setting: Perth, Western Australia.

Participants: A subgroup of 707 eligible mother-fetus pairs from a cohort of 2876 pregnant women and their offspring. The number of mother-fetus pairs varied at each gestational age and by measurement of fetal dimension. Subsequent blood pressure recordings were obtained on approximately 300 of the offspring at age 6 years.

Main results: The findings confirmed the inverse association between birth weight and systolic blood pressure at age 6 . There was, also, an inverse relation between fetal femur length and systolic blood pressure at age 6, adjusted for current height. Furthermore, an inverse association was demonstrated between a statistically derived measure of fetal growth (conditional $z$ score) between 18 and 38 weeks gestation and later systolic blood pressure at age 6 . The effect sizes for all three relations were in the order of 1-2 $\mathrm{mm} \mathrm{Hg}$ per standard deviation change.

Conclusion: The mechanisms underpinning the "fetal origins" hypothesis may be operative early in pregnancy and may be reflected in the length of the fetal femur in early to mid-pregnancy.
\end{abstract}

T he inverse association between birth weight and later blood pressure is firmly established and has, to a large extent, underpinned research into the fetal origins of cardiovascular disease..$^{1-5}$ Implicit in the current expression of the "fetal origins hypothesis" is that retrospective measurements obtained at birth reflect exposure to a suboptimal intrauterine environment, in particular fetal under-nutrition in the second and third trimesters of pregnancy, which subsequently imposes a reduction in fetal growth. A recent meta-analysis describing the relation between blood pressure with birth weight, however, reported that for measurements other than birth weight, the most consistent finding was an inverse association between head circumference and blood pressure. Associations between ponderal index or birth length and systolic blood pressure were absent and there were no consistent relations between systolic blood pressure and gestational age, placental weight, placental to birth weight ratio, or chest circumference. ${ }^{6}$ We have reported that the relation between birth weight and blood pressure in childhood may need to be qualified on the basis of maternal factors known to influence birth weight, such as cigarette smoking during pregnancy. ${ }^{7}$ Furthermore, birth weight is acknowledged as only a crude summary measure of intrauterine growth; the same birth weight may be the outcome of several different paths of growth. ${ }^{8}$ The mechanism by which the growth of a fetus falls from say the 90th centile at a given gestational age to the 30th at a later gestation is likely to differ to that which causes a fetus to maintain a position on the 5 th centile (who is consequently delivered as a "low birth weight" or "growth restricted" baby by conventional definitions). Clearly the first mechanism is more likely to reflect a pathological process and illustrates the need for researchers in this field to avoid confusing fetal growth with fetal size. The emphasis, therefore, needs to shift from proxy measures of intrauterine growth obtained at birth to actual measurements obtained on developing fetuses as recommended by Altman and Hytten in 1989, ${ }^{9}$ and by the Royal College of Obstetricians and Gynaecologists in 1999. ${ }^{10}$

We recently published statistical measures of fetal growth obtained using serial ultrasound and related growth in fetal abdominal circumference to systolic blood pressure in children from the same cohort at 6 years of age. Fetuses whose summary measures indicated poor growth in abdominal circumference had higher blood pressure in early childhood, broadly supporting the "fetal origins hypothesis". ${ }^{11}$ The specific aim of the present analysis was to more clearly elucidate the mechanisms underlying the intrauterine programming of disease by delineating the relative roles of fetal biometry obtained by ultrasound, statistical measures of fetal growth, and birth weight on later systolic blood pressure at age 6.

\section{METHODS}

The current project involved analysis of data from a subgroup of the Western Australian Pregnancy Cohort (Raine) Study. The study was designed, in the first instance, as a randomised controlled trial of the use of multiple ultrasound assessments to improve pregnancy outcome. Briefly, 2876 mothers registering for antenatal care were enrolled at 16-18 weeks gestation at the King Edward Memorial Hospital, the sole tertiary level referral centre for the state of Western Australia. Their selection is detailed elsewhere. ${ }^{12}$ In the "intervention" group $(n=1415)$, pregnancies were monitored by ultrasound imaging, including measurement of head circumference, abdominal circumference, and femur length under standardised conditions, ${ }^{13}$ at approximately 18, 24, 28, 34, and 38 weeks.

Abbreviations: $\mathrm{HC}$, head circumference; $A C$, abdominal circumference; $\mathrm{FL}$, femur length 
Table 1 The effect of paternal and maternal influences on the length of the fetal femur at 24 weeks gestation. (Parameter estimates (95\% confidence intervals) are shown for the increase/decrease in femur length at 24 weeks gestation, by gender, for a one standard deviation increase in separate paternal and maternal characteristics)

\begin{tabular}{lll}
\hline & \multicolumn{2}{l}{ Femur length at 24 weeks } \\
\cline { 2 - 3 } & Females $(\mathrm{n}=270)$ & Males $(\mathrm{n}=297)$ \\
\hline Mean $(\mathrm{SD})(\mathrm{mm})$ & $43.42(2.64)$ & $43.02(2.53)$ \\
& & \\
Paternal characteristics & $0.56(0.14 \text { to } 0.91)^{*}$ & $0.42(0.14 \text { to } 0.70)^{*}$ \\
$\quad$ Height $(\mathrm{cm})$ & $-0.35(-0.80$ to 0.10$)$ & $-0.18(-0.56$ to 0.10$)$ \\
BMl $\left(\mathrm{kg} / \mathrm{m}^{2}\right)$ & $-0.04(-0.32$ to 0.24$)$ & $-0.45(-0.75 \text { to }-0.15)^{*}$ \\
Socioeconomic status $\dagger$ & & \\
Maternal characteristics & $-0.8(-1.4 \text { to }-0.2)^{*}$ & $<0.1(-1.2$ to 1.2$)$ \\
Height $(\mathrm{cm})$ & $-0.03(-0.3$ to 0.24$)$ & $-0.18(-0.36$ to 0.07$)$ \\
BMl $\left(\mathrm{kg} / \mathrm{m}^{2}\right)$ & $-0.1(-0.7$ to 1.0$)$ & $-0.5(-0.2$ to 1.2$)$ \\
Smoker & $0.2(-0.6$ to 0.6$)$ & $0.4(0.1 \text { to } 0.7)^{*}$ \\
Age $(\mathrm{y})$ & $-0.32(-0.64 \text { to }<0.1)^{*}$ & $-0.18(-0.46$ to 0.10$)$ \\
Haemoglobin $(\mathrm{g} / \mathrm{dll})$ & $0.02(-5.2$ to 5.2$)$ & $-0.60(-5.6$ to 4.5$)$ \\
Parity $>2$ & & \\
\hline †Composite index: $0=$ highest value, $4=$ lowest. * $\mathrm{p}<0.05$. & \\
\hline
\end{tabular}

Concurrently, the study was designed as a prospective cohort study of the influence of maternal, intrauterine and perinatal influences on subsequent health, with an a priori decision to focus on different systems at different review ages of the offspring. ${ }^{14-17}$ Approval was obtained from the relevant institutional ethics committee and written consent was obtained from each of the participants. Socioeconomic status was calculated from a composite score based on maternal and paternal occupation, highest level of education attained, and total income.

A follow up assessment was performed on the children at age 6 at the Institute for Child Health Research. Blood pressure was recorded using a Dinamap 8100 semi-automated oscillometric monitor (Critikon, Arlington, Texas, United States), under standardised conditions, reported in detail elsewhere. ${ }^{18}$ The cuff size was dictated by the arm size, which ensured that the cuff width to arm circumference ratio of $40 \%-50 \%$ was met as recommended by the American Heart Association. ${ }^{19}$ Calibration of the instrument revealed there had been no measurement drift throughout the period of the study. These machines correlate closely with intra-arterial pressures and the mean error is smaller than with conventional auscultatory methods. ${ }^{20}$ The average of two readings taken one minute apart was used in the analysis. We confine our report to analyses involving systolic blood pressure because the association between diastolic blood pressure and birth weight is consistently reported as weaker and less consistent than that with systolic blood pressure and birth weight. Furthermore, the Dinamap 8100 is associated with an artefact when used to determine diastolic blood pressure. ${ }^{21}$ All data were computerised in SAS format (SAS, Cary, North Carolina).

Subsequent analyses for the current project were confined to the 1276 singleton, term (37-42 weeks completed gestation) births in the intervention group. From these, pregnancies complicated by congenital abnormalities $(n=18)$, diabetes $(n=55)$, proteinuric hypertension $(n=31)$, and antepartum haemorrhage $(n=119)$ were excluded because of the possible confounding (or indeed effect modifying) effects of an intrinsic maternal or fetal disorder on growth. Data were also excluded if there was a discordance of more than seven days between the date of the last menstrual period and an assessment of gestation determined by ultrasound at enrolment $(n=346)$. These exclusions resulted in 707 eligible mother/fetus pairs on whom a total of 3450 ultrasound measurement occasions were performed between 15 and 42 weeks gestational age.
Based on serial biometric data from the 707 eligible fetuses, we developed statistical models of the relation between the distribution of three fetal dimensions: head circumference (HC), abdominal circumference (AC) and femur length (FL) and gestational age described in detail elsewhere. ${ }^{11}$ These models were used to motivate three summary measures of individual fetal growth, namely, (1) the conditional centile or $\mathrm{z}$ score of a current measurement given an earlier value on the same measurement (as proposed by Royston ${ }^{22}$ ); (2) the best linear unbiased predictor of the subject specific growth gradient on a transformed scale; and (3) the standardised residual at a given later gestational age. The conditional z score compares an observed measurement made at a later time, say 38 weeks gestation, with the theoretical distribution of all measurements made at 38 weeks on fetuses who had the same measured value at an earlier time. For example, consider two fetuses who at 18 weeks gestation both lie on the 25 th centile of the population distribution for a given fetal dimension. At 38 weeks one may have progressed to the 75 th population centile, whereas the other may have remained at the 25 th centile. The latter fetus would have a conditional centile close to the 50th as he or she has maintained their population centile. The other fetus would, however, have a high conditional centile, as not many fetuses on the 25 th population centile at 18 weeks will progress to the 75th population centile at 38 weeks. We can therefore say that this fetus had a faster growth velocity when compared with the other. The conditional $\mathrm{z}$ score, therefore, focuses on changes within the individual and provides a quantitative answer to the question "has the fetus shown fast or slow growth to the current gestational age when judged against an earlier measurement". We confine our report to this measure as our summary measure of fetal growth velocity between 18 and 38 weeks gestation.

Linear regression was used to relate systolic blood pressure at age 6 to the observed measurement of each fetal dimension obtained within one week of the nominal gestational ages of $18,24,28,34$, and 38 weeks from the 707 mother/fetus pairs that satisfied the above selection criteria.

The simultaneous relations between systolic blood pressure at age 6 (adjusted for current weight), birth weight, and a summary measure of fetal growth were examined by determining mean values for systolic blood pressure at age 6 in each of the cells of a two way contingency table, defined by tertiles of birth weight and tertiles of conditional z scores. This procedure was performed separately for each of the three fetal dimensions $\mathrm{HC}, \mathrm{AC}$, and FL. While every effort was made to 


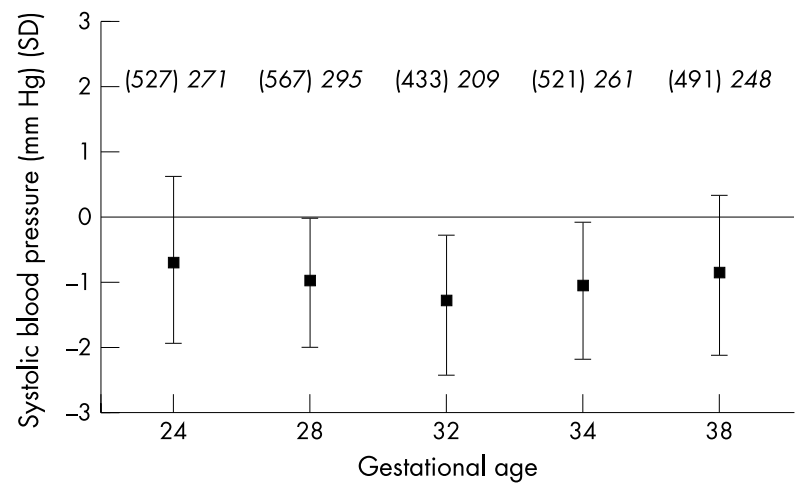

Figure 1 Parameter estimates and $95 \% \mathrm{Cl}$ for the decrease in systolic blood pressure at age 6 associated with a one standard deviation increase in femur length by gestational age, with adjustment for current height (numbers in parentheses = number of measurements obtained at each gestational age, numbers in italics are those with subsequent blood pressure measurements).

follow up all 707 eligible mother-child pairs, the number of children with blood pressure measurements at age 6 included in this analysis was considerably less than this. The reasons underlying this apparent high rate of attrition were more complex than the losses to follow up commonly associated with large, longitudinal studies. For example, it was not possible to record an acceptable measurement for every fetal dimension at a single scan. For example, the length of the fetal femur was recorded in 567 women at 24 weeks gestation. Of these 567 women, 378 attended with their offspring for follow up physical assessments at age 6 (the overall physical assessment follow up rate for the entire cohort of children was 65\%). Blood pressure recordings were missing on 11 of these children. Furthermore, measurements obtained from children classified as uncooperative $(n=72)$ were also excluded. Distress caused by inflation of the cuff or general restlessness were the commonest causes of being classified as uncooperative as would be anticipated in such a young age group. What we considered as valid blood pressure measurements were, therefore, obtained on 139 female and 156 male offspring from the original 707 eligible mother/fetus pairs. The values determining the tertiles of birth weight differ for each fetal dimension. This is a consequence of using the observed birth weight distribution of fetuses that had valid measurements for that fetal dimension. The alternative would have been to define tertiles according to the cohort birth weight distribution, which potentially has the disadvantage of generating small, and thus unrepresentative, samples in the extreme cells of the contingency table.

\section{RESULTS}

The mean (SD) age of the participants at follow up was 5.88 (0.17) years. The mean (SD) systolic blood pressure at age 6 was not statistically significantly different between genders (104.6 (8.1) $\mathrm{mm} \mathrm{Hg}$ in females and 104.4 (6.8) $\mathrm{mm} \mathrm{Hg}$ in males $(95 \% \mathrm{CI}(-1.7$ to 2.1$)$ for the difference between the means)). Systolic blood pressure at age 6, therefore, was not adjusted for gender. The mean birth length was $49.4(2.1) \mathrm{cm}$. There was a statistically significant inverse relation between birth length and systolic blood pressure at age 6, adjusted for current height $(-0.6$ (95\% CI -1.0 to -0.2$) \mathrm{mm} \mathrm{Hg}$ per standard deviation increase in birth length).

There were inverse relations between femur length, adjusted for current height, and systolic blood pressure at age 6 years (fig 1) with an effect size of $1-2 \mathrm{~mm} \mathrm{Hg}$ per standard deviation change in femur length. Adjustment was made for current height so as not to obscure a possible negative relation between blood pressure and femur length. Similar relations existed when adjustment was made for current weight or current body mass index. The earliest age at which this relation was statistically significant was 24 weeks gestation. Significant univariate relations were identified between femur length at 24 weeks gestation and paternal height, maternal age, and socioeconomic status in males; and paternal height, maternal height, and maternal haemoglobin in females (table 1). Neither head nor abdominal circumference or their ratio, at any gestational age, was significantly associated with systolic blood pressure at age 6 .

Table 2 Simultaneous effects of birth weight and relative growth rate (determined by tertiles of conditional z scores) of the fetal (a) AC, (b) FL, and (c) HC on systolic blood pressure $(\mathrm{mm} \mathrm{Hg})$ at age 6 , adjusted for current weight. Numbers in each tertile appear in parentheses; numbers of those with valid blood pressure recordings appear in italics in square brackets)

\begin{tabular}{|c|c|c|c|c|}
\hline Growth & Slow (163) & Medium (161) & Fast (167) & Total (491) \\
\hline \multicolumn{5}{|l|}{$\begin{array}{l}\text { (a) AC } \\
\text { Birth weight (kg) }\end{array}$} \\
\hline$<3.3(157)$ & 106.2 [39] & $104.6[17]$ & 109.0 [10] & $106.6[66]$ \\
\hline $3.3-3.7(173)$ & $104.7[27]$ & $104.0[33]$ & $104.8[20]$ & $104.5[80]$ \\
\hline$>3.7$ (161) & $103.8[9]$ & $102.2[20]$ & $102.4[44]$ & $102.6[73]$ \\
\hline Total (491) & 104.9 [75] & $103.6[70]$ & $105.4[74]$ & 104.7 [219] \\
\hline Growth & Slow (172) & Medium (156) & Fast (178) & Total (506) \\
\hline \multicolumn{5}{|l|}{ (b) FL } \\
\hline$<3.5$ (171) & 105.0 [19] & 106.7 [24] & $106.9[26]$ & 106.2 [69] \\
\hline $3.5-3.7(165)$ & 107.4 [39] & $103.3[26]$ & $103.4[13]$ & $104.7[78]$ \\
\hline$>3.7$ (170) & $101.4[17]$ & $104.0[23]$ & $102.6[42]$ & $101.0[83]$ \\
\hline Total (506) & $104.6[76]$ & $104.0[73]$ & $104.3[81]$ & 104.1 [230] \\
\hline Growth & Slow (136) & Medium (123) & Fast (134) & Total (393) \\
\hline \multirow{2}{*}{\multicolumn{5}{|c|}{$\begin{array}{l}\text { (c) } \mathrm{HC} \\
\text { Birth weight }(\mathrm{kg})\end{array}$}} \\
\hline & & & & \\
\hline$<3.4(135)$ & $106.0[30]$ & $109.7[13]$ & 104.2 [12] & $106.6[55]$ \\
\hline $3.4-3.7$ (127) & $101.2[16]$ & $105.9[25]$ & $105.2[17]$ & 104.1 [59] \\
\hline$>3.7(131)$ & $103.0[16]$ & 101.4 [19] & 102.1 [32] & $102.2[66]$ \\
\hline Total (393) & 103.4 [62] & $105.7[57]$ & $103.8[61]$ & $104.3[180]$ \\
\hline
\end{tabular}


Table 2 illustrates the interplay between attained birth weight, and fetal growth between 18 and 38 weeks gestation in determining subsequent systolic blood pressure at age 6 . The inverse association between birth weight and systolic blood pressure was replicated in each of the samples defined by each fetal dimension. For AC, a difference between the means for the highest and lowest birth weight groups of -3.8 (95\% CI (-7.1 to -0.6)) $\mathrm{mm} \mathrm{Hg}$ ) was observed. Similar results were observed for the other two fetal dimensions. The highest systolic blood pressure (109.0 mm Hg) occurred in the group of babies born lightest but who had a fast AC growth rate.

For FL, the lowest systolic blood pressures (101.4 mm Hg) were found in the heaviest babies whose femurs grew at the slowest rate. There was a statistically significant difference between the means of that systolic blood pressure and that of the corresponding extreme group of lower birthweight babies with fast growing femurs for whom the mean systolic blood pressure was $106.2 \mathrm{~mm} \mathrm{Hg}$ (difference between the means $-5.5(-10.9$ to -0.1$) \mathrm{mm} \mathrm{Hg})$.

There were no consistent patterns in the inter-relations between growth in head circumference, birth weight, and later systolic blood pressure.

The results of the analyses based on the contingency tables were confirmed by linear regression modelling. The regression coefficients (standard error) for a one standard deviation change in fetal growth velocity (as defined by the conditional z scores) on systolic blood pressure at age 6 , adjusted for current weight, were $-0.60(0.6),-0.7(0.5)$, and $-0.4(0.6) \mathrm{mm}$ $\mathrm{Hg}$ for AC, FL, and $\mathrm{HC}$, respectively.

\section{DISCUSSION}

In this project we examined a body of serial ultrasound data obtained on developing fetuses and related it to a subsequent health outcome, namely, systolic blood pressure at age 6 . We classified individuals by three estimates of fetal growth or size: biometry of FL, AC, and HC, at each of five gestational ages; a statistical model of fetal growth between 18 and 38 weeks gestation; and birth weight.

We demonstrated that the length of the fetal femur at 24 weeks gestation, adjusted for current height, was statistically significantly related to subsequent systolic blood pressure at age 6. The effect size for this relation was comparable to that which we found between birth weight and systolic blood pressure at age 6, when the latter relation was adjusted for current weight. ${ }^{7}$ This relation in turn was comparable to that observed between birth weight and systolic blood pressure in the 1996 meta-analysis of Law and Shiell, ${ }^{4}$ namely 2-3 mm Hg per kg change in birth weight. There were gender differences in the determinants of the length of the fetal femur in mid-gestation. Analysis of body proportions in a Finnish cohort concluded that the paths of intrauterine growth that led to low birth weight and later coronary heart disease differed between the sexes. ${ }^{23}$ We demonstrated a negative association between femur length at 24 weeks gestation and maternal height, in females. This finding was interesting in that it may indicate that early femur length in utero does not reflect genetically determined height.

We delineated the relation between the conditional z score (as a measure of fetal growth) and later systolic blood pressure. A $1 \mathrm{~kg}$ change in birth weight constitutes at least a two standard deviations change on a population basis. We, therefore, related a two standard deviations increase in conditional z scores for AC, FL, and HC to later systolic blood pressure at age 6 and determined effect sizes of $-1.2,-1.4$, and -0.8 $\mathrm{mm} \mathrm{Hg}$, respectively. These relations, while not statistically significant, their effect sizes are comparable to that observed in the more recent meta-analysis of the relation between birth weight and systolic blood pressure, ${ }^{6}$ namely $\mathrm{l}-2 \mathrm{~mm} \mathrm{Hg}$ per $\mathrm{kg}$ change in birth weight. This may be interpreted as supporting the "fetal origins hypothesis" in its current interpretation in

\section{Key points}

- We confirmed the acknowledged inverse relation between birth weight and later systolic blood pressure.

- Growth of the fetal femur and abdominal circumference in the second and third trimester of pregnancy have similar effect sizes, when related to systolic blood pressure at age 6.

- The length of the fetal femur at 24 weeks gestation was statistically significantly inversely related to systolic blood pressure at age 6 , with an effect size similar to that of birth weight and systolic blood pressure at age 6

- The mechanisms underpinning the "fetal origins" hypothesis may be operative early in pregnancy and may be reflected in the length of the fetal femur in early-mid pregnancy.

that intrauterine growth in mid and late gestation, was inversely related to systolic blood pressure at age 6 .

We delineated the simultaneous relations between systolic blood pressure at age 6 (adjusted for current weight), birth weight, and a summary measure of fetal growth for each of the three dimensions AC, FL, and HC. We adjusted systolic blood pressure at age 6 for current weight because of the concurrent positive associations between this variable and systolic blood pressure and birth weight. Adjustment for current weight serves to highlight the relation between birth weight and blood pressure in childhood and we have argued that birth weight, rather than birth weight adjusted for current weight, is still the relevant predictor of later blood pressure within the context of the "fetal origins hypothesis". ${ }^{24}$ To challenge the "fetal origins hypothesis" in its current definition we sought to determine if the attainment of a given birth weight and its subsequent association with later blood pressure was a reflection of fetal growth in mid-late gestation. At later gestational ages, $\mathrm{AC}$ is the most powerful biometric descriptor of growth and reflects both liver size and subcutaneous fat, each of which is a sensitive marker of fetal nutrition. ${ }^{13}$ For a given birth weight, an increasing growth rate of the AC was not associated with a decrease in systolic blood pressure, either overall, or within tertiles of birthweight groups. The highest systolic blood pressure occurred in the group of relatively low birthweight babies who had a fast AC growth rate. This may reflect a slow first half growth rate and later catch up or may reflect the growth of different organs. At 18 weeks of pregnancy the adrenal glands are among the largest intra-abdominal organs. Factors that influence growth of the adrenal glands in early pregnancy may be important in setting size at the beginning of our growth measures. Later other organs, liver, and subcutaneous fat may be more important. Of note, the results presented are based on measures of growth determined after 18 weeks gestation. Interpretation, therefore, is subject to the caveat that the fetus has not experienced growth restriction prior to 18 weeks gestation, when the first ultrasound scan is conducted. It would be expected that the lowest blood pressures would be in infants with medium growth rates who have grown appropriately for their size at 18 weeks. Our finding that for AC, infants with medium growth rates have the lowest blood pressure within each birth weight group is in keeping with this expectation. Similarly, the lowest systolic blood pressures were found in those babies who were heaviest and who exhibited medium or fast AC growth rates.

The consistent inverse relation between birth weight and systolic blood pressure within AC growth groups does not, however, support the argument that the relation between systolic blood pressure and low birth weight is a consequence of fetal under-nutrition associated with a slow growing AC in mid-late gestation. Doyle et al have shown a dose response relation between nutrient intake and birth weight up to 3270 $\mathrm{g}$ in a socially disadvantaged population in London. ${ }^{25}$ They also demonstrated in a randomised controlled trial that supplementation with a broad based nutritional supplement starting 
in the second trimester failed to show a reduction in the incidence of low birth weight. ${ }^{26}$ Programmes of nutritional intervention both pre-conception and during the first trimester with low income women in the United States, by comparison, have shown a reduction in the incidence of low birth weight. ${ }^{27}$ These studies, coupled with our report, have public health implications in that if low birth weight does predispose to later disease in adulthood, maternal diet in the first trimester may be the crucial factor.

For FL, the lowest systolic blood pressures were found in the heaviest babies whose femurs grew at the slowest rate and there was a statistically significant difference between the means of the extreme groups of combinations of birth weight and FL growth rate. Animal studies have shown that food restriction throughout pregnancy causes disproportionate growth with relative preservation of the brain, restriction of liver growth, and a reduction in skeletal mass. ${ }^{28}$ Further animal studies have shown that the effects of a constrained materno-placental supply of nutrients are critically dependent on fetal nutrient requirements. ${ }^{29}$ Maternal under-nutrition during late pregnancy slowed the growth rate of rapidly growing sheep fetuses with high requirements, while having little effect on those growing more slowly. ${ }^{30}$ The magnitude of fetal demand for nutrients is greatest late in pregnancy and is primarily determined by genetic and environmental effects on the trajectory of fetal growth set at an early stage of development. ${ }^{31}$ Therefore, the length of the fetal femur at 24 weeks gestation may reflect adaptations in fetal growth to influences such as paternal height, maternal age and height, socioeconomic status, and maternal haemoglobin. These adaptations determine the fetus's subsequent growth rate and consequently its requirements for nutrients in advance of its greatest demand for nutrients. The mechanisms, therefore, underpinning the growth restriction that predisposes to adult disease may be operative well before third trimester growth. Our findings support those of a recent publication in which the authors also suggested that the initiating events of blood pressure programming occur early in pregnancy. ${ }^{32}$

We studied a cohort of relatively well nourished, affluent, racially homogenous ( $>90 \%$ white) individuals, as reflected in a mean birth weight of $3512(\mathrm{SD}=457) \mathrm{g}$. We related three separate markers of fetal growth/size to subsequent systolic blood pressure and determined remarkably similar effect sizes, in the order of $1-2 \mathrm{~mm} \mathrm{Hg}$. These effect sizes were also remarkably similar to those obtained in 80 studies in diverse populations in terms of race, gender, age, affluence, and nourishment. ${ }^{6}$ This would tend to suggest that the relation between intrauterine growth while consistent, is small and may be attributable to a variety of mechanisms, which differ among populations. This should be taken into account when discussing the relative importance of intrauterine growth on subsequent blood pressure.

Of note, we were of the opinion that the sample size was sufficiently large to allow us to apply strict criteria for inclusion in further analyses to the sample of fetuses with repeated ultrasound measurements. We did not repeat these analyses in the overall sample with serial ultrasound measures because that sample would include fetuses that might be growth retarded because of intrinsic maternal or fetal anomalies and also fetuses that might appear to be growth retarded as a consequence of inaccurate estimation of gestational age. We explored, therefore, the relations between measures of fetal size and fetal growth to later blood pressure in a sample of fetuses that were determined to be as "normal" as possible, and whose gestational age was estimated accurately using both the mother's recall and measurements determined by ultrasound.

We have shown direct evidence that intrauterine growth is related to a subsequent health outcome, in this case systolic blood pressure at age 6 . Our results suggesting that the fetus adapts relatively early in pregnancy in advance of future demands for nutrients are consistent with recent observations in human and animal studies. The correlation of serial ultrasound biometry to later blood pressure in the same individuals is a unique contribution to the level of knowledge on this issue.

\section{ACKNOWLEDGEMENTS}

Thanks to the staff at The King Edward Memorial Hospital and The Women and Infants Research Foundation involved with The Western Australian Pregnancy Cohort Study. Particular acknowledgement also to Garth Kendall, Lee Clohessy, Karen Moonen, Carolyn Smargiassi and Meryl Biggs at the TVW Telethon Institute for Child Health Research. We acknowledge with gratitude the women and families who participated in the study.

\section{Authors' affiliations}

K V Blake, J P Newnham, Department of Obstetrics and Gynaecology, The University of Western Australia, Subiaco, Western Australia

L C Gurrin, J P Newnham , Women and Infants Research Foundation, King Edward Memorial Hospital, Subiaco, Western Australia

K V Blake, L J Beilin, Department of Medicine, The University of Western Australia, Perth

F J Stanley, G E Kendall, TVW Telethon Institute for Child Health Research, West Perth, Western Australia

L I Landau, The University of Western Australia Faculty of Medicine and Dentistry, Queen Elizabeth II Medical Centre, Nedlands, Western Australia

Funding: We are grateful for the financial support of the Raine Research Foundation of The University of Western Australia and the National Health and Medical Research Council of Australia, Project Grant number: 930745. Dr Kevin V Blake is a recipient of a Public Health Research Development Committee Medical Postgraduate Scholarship from the National Health and Medical Research Council of Australia.

Conflicts of interest: none.

\section{REFERENCES}

1 Barker DJP. Fetal origins of coronary heart disease. BM 1995;311:171-4

2 Lucas A. Role of nutritional programming in determining adult morbidity. Arch Dis Child 1994:71:288-90.

3 Law CM, de Swiet M, Osmond C, et al. Initiation of hypertension in utero and amplification throughout life. BN 1993;306:24-7

4 Law CM, Shiell AW. Is blood pressure inversely related to birth weight? the strength of evidence from a systematic review of the literature. J Hyperten 1996;14:935-41.

5 Whincup PH, Cook DG, Papacosta O. Do maternal and intrauterine factors influence blood pressure in childhood? Arch Dis Child 1992;67:1423-9

6 Huxley RR, Shiell AW, Law CM. The role of size at birth and postnatal catch-up growth in determining systolic blood pressure: a systematic review of the literature. J Hyperten 2000;18:815-31.

7 Blake KV, Gurrin LC, Evans SF, et al. Maternal cigarette smoking during pregnancy, low birth weight and blood pressure in early childhood. Early Hum Devel 2000;57:137-47.

8 Harding JE, Johnston BM. Nutrition and fetal growth. Reprod Fertil Dev 1995; 7:539-47.

9 Altman DG, Hytten FE. Intrauterine growth retardation: Lets be clear about it. BrJ Obstet Gynaecol 1989;96:1 127-32.

10 36th RCOG Study Group. Recommendations arising from the $36^{\text {th }}$ RCOG Study Group. In: Shaughn O'Brien PM, Wheeler T, et al, eds. Fetal programming: influences on development and disease in later life. London: RCOG Press, 1999:463-5

11 Gurrin LC, Blake KV, Evans SF, et al. Statistical measures of fetal growth using linear mixed models applied to the fetal origins hypothesis. Stat Med 2001;20:3391-409.

12 Newnham JP, Evans SF, Michael CA, et al. Effects of frequent ultrasound during pregnancy: a randomised controlled trial. Lancet 1993;342:887-91.

13 Newnham JP, Evans SF. Fetal biometry. In: Rodeck CH, Whittle M, eds. Fetal medicine: basic science and clinical practice. London: Churchill Livingstone, 1999:939-53.

14 Williams LA, Evans SF, Newnham JP. Prospective cohort study of factors influencing the relative weights of the placenta and the newborn infant. BM 1997:314:1864-8.

15 Stick SM, Burton PR, Gurrin L, et al. Effects of maternal smoking during pregnancy and a family history of asthma on respiratory function in newborn infants. Lancet 1996;348:1060-4.

16 Oddy WH, Holt PG, Sly PD, et al. Association between breast-feeding and asthma in 6-year-old children: findings of a prospective birth cohort study. BM 1999;319:815-19. 
17 Blake KV, Gurrin LC, Beilin L, et al. Placental weight and placental ratio as predictors of later blood pressure in childhood. J Hyperten 2001;19:1-6.

18 Blake KV, Gurrin LC, Evans SF, et al. Reference ranges for blood pressure in pre-school Australians, obtained by oscillometry. J Paediatr Child Health 2000;36:41-6.

19 Frolich ED, Grim C, Labarthe DR, et al. Recommendations for human blood pressure determinations by sphygmomanometers. Report of a special task force appointed by the steering committee, American Heart Association. Circulation 1988;77:502-14A.

20 Park MK, Menard SM. Accuracy of blood pressure measurement by the Dinamap monitor in infants and children. Pediatrics 1987;79:907-14

21 O'Brien E, Atkins N. Inaccuracy of the Dinamap 8100 portable monitor. Lancet 1997;349:1026.

22 Royston P. Calculation of unconditional and conditional reference intervals for foetal size and growth from longitudinal measurements. Stat Med 1995;14:1417-36.

23 Forsen T, Eriksson JG, Tuomilehto J, et al. Growth in utero and during childhood among women who develop coronary heart disease: longitudinal study. BM 1999;319:1403-7.

24 Blake KV, Gurrin LC, Evans SF, et al. Adjustment for current weight and the relationship between birth weight and blood pressure in childhood. $J$ Hyperten 2000; 18:1007-12.

25 Doyle W, Crawford MA, Wynn AHA, et al. The association between maternal diet and birth dimensions. J Nutr Med 1990;1:9-17.
26 Doyle W, Wynn AHA, Crawford MA, et al. Nutritional counselling and supplementation in the second and third trimester of pregnancy, a study in a London population. J Nutr Med 1992;3:249-56.

27 Scholl TO, Hediger ML, Bendich A, et al. Use of prenatal supplements: influence on the outcome of pregnancy. Am J Epidemiol 1997; 146:134-41

28 Robinson JS, McMillen C, Edwards L, et al. The effect of maternal nutrition on growth and development before and after birth. In: Shaughn O'Brien PM, Wheeler T, Barker DJP, eds. Fetal programming: influences on development and disease in later life. London: RCOG Press, 1999:217-30

29 Harding JE, Liu L, Evans P, et al. Intrauterine feeding of the growth-retarded fetus: can we help? Early Hum Devel 1992;29:193-7.

30 Harding JE. Prior growth rate determines the fetal response to acute maternal undernutrition in fetal sheep of late gestation. Prenatal and Neonatal Medicine 1997;2:300-9.

31 Godfrey KM, Brier BH, Cooper C. Constraint of the materno-placental supply of nutrients: causes and consequences. In: Shaughn O'Brien PM, Wheeler T, Barker DJP, eds. Fetal programming: influences on development and disease in later life. London: RCOG Press, 1999:283-98

32 Thame M, Osmond C, Wilks R, et al. Blood pressure is related to placental volume and birth weight. Hypertension 2000;35:662-7. 